Non-renormalization conditions for four-gluon scattering in supersymmetric string and field theory

This content has been downloaded from IOPscience. Please scroll down to see the full text. JHEP11(2009)063

(http://iopscience.iop.org/1126-6708/2009/11/063)

View the table of contents for this issue, or go to the journal homepage for more

Download details:

IP Address: 186.217.236.119

This content was downloaded on 25/02/2015 at $18: 42$

Please note that terms and conditions apply. 


\title{
Non-renormalization conditions for four-gluon scattering in supersymmetric string and field theory
}

\author{
Nathan Berkovits, ${ }^{a}$ Michael B. Green, ${ }^{b}$ Jorge G. Russo ${ }^{c, d}$ and Pierre Vanhove ${ }^{e, f}$ \\ ${ }^{a}$ Instituto de Física Teórica, UNESP-Universidade Estadual Paulista, \\ R. Dr. Bento T. Ferraz 271, Bloco II, Sao Paulo, SP 01140-070, Brasil \\ ${ }^{b} D A M T P$, \\ Wilberforce Road, Cambridge CB3 0WA, U.K. \\ ${ }^{c}$ Institució Catalana de Recerca i Estudis Avançats (ICREA), \\ Departament ECM and Institut de Ciencies del Cosmos, \\ Facultat de Fisica, University de Barcelona, \\ Av. Diagonal, 647, Barcelona 08028 Spain \\ e IHES, Le Bois-Marie, \\ 35 route de Chartres, F-91440 Bures-sur-Yvette, France \\ ${ }^{f}$ Institut de Physique Théorique, CEA/Saclay, \\ F-91191 Gif-sur-Yvette, France \\ E-mail: nathan.berkovits@gmail.com, M.B.Green@damtp.cam.ac.uk, \\ jrusso@ub.edu, pierre.vanhove@cea.fr
}

ABSTRACT: The constraints imposed by maximal supersymmetry on multi-loop contributions to the scattering of four open superstrings in the $\mathrm{U}(N)$ theory are examined by use of the pure spinor formalism. The double-trace term $k^{2} t_{8}\left(\operatorname{tr} F^{2}\right)^{2}$ (where $k$ represents an external momentum and $F$ the Yang-Mills field strength) only receives contributions from $L \leq 2$ (where $L$ is the loop number) while the single-trace term $k^{2} t_{8}\left(\operatorname{tr} F^{4}\right)$ receives contributions from all $L$. These statements are verified up to $L=5$, but arguments based on supersymmetry suggest they extend to all $L$. This explains why the single-trace contributions to low energy maximally supersymmetric Yang-Mills field theory are more divergent in the ultraviolet than the double-trace contributions. We also comment further on the constraints on closed string amplitudes and their implications for ultraviolet divergences in $\mathcal{N}=8$ supergravity.

KEywords: Extended Supersymmetry, Gauge Symmetry, Field Theories in Higher Dimensions

ARXIV EPRINT: 0908.1923 


\section{Contents}

1 Introduction 1

1.1 General properties of the four-gluon amplitude 2

1.2 Organization of the paper 3

2 Open-string scattering amplitudes in the pure spinor formalism 4

2.1 The multi-loop functional integral for world sheets with $B$ boundaries $\quad 5$

$\begin{array}{lll}2.2 & \text { The open-string vertex operator } & 7\end{array}$

3 The scattering of four open strings $\quad 8$

3.1 Zero-mode integrals and momentum prefactors 9

$\begin{array}{lll}3.2 & \text { Inverse derivative factors } & 10\end{array}$

4 Contributions of handles $\quad 14$

5 Connections with maximal SYM theory in various dimensions $\quad \mathbf{1 5}$

$\begin{array}{lll}5.1 \text { Onset of ultraviolet divergences in various dimensions } & 16\end{array}$

$\begin{array}{lll}6 & \text { Summary and comments on higher-point amplitudes } & 17\end{array}$

\section{Introduction}

Supersymmetry imposes crucial constraints on the structure of scattering amplitudes in supersymmetric gauge and gravitational theories, which generally leads to a moderation of ultraviolet divergences. These constraints are particularly strong for maximally supersymmetric theories, which are difficult to analyse using conventional superspace techniques due to the absence of an off-shell superspace formalism. However, it is possible to analyse such field theory supersymmetry constraints by considering the low energy limit of the corresponding open or closed superstring theories. In particular, the pure spinor formalism $[1,2]$ is a framework for constructing multi-loop string theory amplitudes in a manner that preserves all the space-time supersymmetries. An example of constraints obtained in this manner comes from the analysis of multi-loop contributions to the four-graviton amplitude in type II superstring theory [3]. These constraints imply that interactions of the form $\partial^{2 k} R^{4}$ (where $R^{4}$ denotes a particular contraction of four Riemann curvatures) do not get any perturbative contributions beyond $k$ loops in the ten-dimensional theory, at least for $k \leq 6$. These conditions follow from the fact that interactions with $k<6$ are $F$-terms that are given by integrals over a fraction of the full 32-component superspace. A striking consequence of this that follows on purely dimensional grounds is that ultraviolet divergences should be absent up to at least nine loops in four-dimensional $(D=4) \mathcal{N}=8$ 
supergravity [4]. By contrast, analyses of counterterms that exploit less than the full $\mathcal{N}=8$ supersymmetry give weaker conditions $[5,6]$.

The main purpose of this paper is to extend these considerations to open string theory and, hence, to its low energy limit — maximally supersymmetric Yang-Mills (SYM) theory.

\subsection{General properties of the four-gluon amplitude}

For simplicity we will consider the case of open strings scattering on $N$ coincident $D p$ branes, for which the world-sheet is orientable and which corresponds to a $\mathrm{U}(N)$ gauge theory in the low energy field theory limit. It has long been known that ultraviolet divergences are absent in maximally supersymmetric Yang-Mills field theory in dimensions $D \leq 4$ to all orders in perturbation theory — one does not even need to exploit the full power of maximal supersymmetry to argue that the theory is UV finite [7-10]. Indeed, there are finite $\mathcal{N}=2$ and $\mathcal{N}=1$ super Yang-Mills theories. It is sufficient to know that the dimension four operator $t_{8} F^{4}$ factors out in the sum of Feynman diagrams at every order in perturbation theory (where $t_{8}$ is a standard eight-index tensor reviewed in appendix 9.A. of [11] that contracts the space-time indices in $F^{4}$ while the contraction of the gauge indices will be discussed later). It follows by simple dimensional analysis that the perturbative contributions are ultraviolet finite at each order (all $L$ ) in dimensions $D \leq 4$. However, the situation is better than that because the CP-even $t_{8} F^{4}$ is related by supersymmetry to the $\mathrm{CP}$-odd anomaly cancelling term $B \wedge F^{4}$ in ten dimensions, which is expected to be one-loop exact [12-21]. This means that the $L>1$ contributions to the string scattering amplitude must have a low energy limit that behaves as $s^{\gamma_{L}} t_{8} F^{4}$ with $\gamma_{L} \geq 1$, so that the prefactor contains at least two extra powers of momentum ${ }^{1}$. We may interpret this contribution as a term of the form $\partial^{2 \gamma_{L}} t_{8} F^{4}$ in the effective action and in the following we will often pass between the amplitude and the effective action without comment. In fact, there are indications that $\gamma_{L}=1$ for $L>1$ from direct perturbative evaluations of the four gluon amplitude in maximally supersymmetric Yang-Mills theory [22-24] or by $\mathcal{N}=3$ superspace arguments in four dimensions [5]. Assuming $\gamma_{L}=1$, it is easy to see using dimensional analysis that an $L$-loop amplitude with a prefactor of $s t_{8} F^{4}$ is ultra-violet finite in dimensions $D<4+6 / L$.

Although extended supersymmetry determines the dynamical prefactor to be of the form $s^{\gamma_{L}} t_{8} F^{4}$ [25], there is also a dependence on the gauge group and on the string coupling constant $g_{s}$, which is related to the Yang-Mills coupling $g_{\mathrm{YM}}$ by $g_{s}=g_{\mathrm{YM}}^{2} / 4 \pi$. For example, for the gauge group $\mathrm{U}(N)$ (which is the simplest example) there are two independent group theory structures in the field theory four-gluon amplitude - a single trace term $t_{8} \operatorname{tr}\left(F^{4}\right)$ and a double-trace $t_{8}\left(\operatorname{tr} F^{2}\right)^{2}$ term, where we are now taking $F$ to be an $N \times N$ matrix in the defining representation of $\mathrm{U}(N)$ and tr denotes a trace on these indices. Contributions to the amplitude of the general form $s^{\gamma_{L}} t_{8} \operatorname{tr}\left(F^{4}\right)$ and $s^{\beta_{L}} t_{8}\left(\operatorname{tr} F^{2}\right)^{2}$ will be constrained by supersymmetry in different manners at a given order in perturbation theory (i.e., for a given power of $g_{s}$ ). Our aim is to determine the values of $\gamma$ and $\beta$ by considering the low energy limit of the four-gluon amplitude in open superstring theory.

\footnotetext{
${ }^{1}$ The factor of $s^{\gamma_{L}}$ in this expression, and all those that follow, is intended to indicate the power of Mandelstam invariants - the precise expression involves a function of $s, t, u$ with a detailed structure that will not concern us here.
} 
Certain properties of the open superstring four-gluon amplitude are well known. For example, at tree-level $(L=0)$ the world-sheet is a disk with all vertex operators describing the external states coupled to the boundary. In this case, in the low energy limit only a single-trace $g_{s}^{-1} t_{8} \operatorname{tr}\left(F^{4}\right)$ term contributes. For $L=1$ the world-sheet is an annulus, which has two boundaries. When all vertex operators are attached to a single boundary there is a low energy contribution that behaves as $N t_{8} \operatorname{tr}\left(F^{4}\right)$ (where the factor of $N$ arises from a trace over the free boundary). There is also an $L=1$ contribution when there is a pair of vertex operators attached to each boundary, which reduces in the low energy limit to terms of the form $t_{8}\left(\operatorname{tr} F^{2}\right)^{2}$. These are $F$-terms that are protected from higher-loop $(L>1)$ quantum corrections. By an $F$-term, we mean a term which cannot be expressed as an integral over $16 \theta$ 's of a gauge-invariant integrand. On the other hand, a $D$ term is a term which can be expressed as an integral over $16 \theta$ 's of a gauge-invariant integrand. We will confirm, using string loop calculations, that $t_{8} \operatorname{tr}\left(F^{4}\right)$ and $t_{8}\left(\operatorname{tr} F^{2}\right)^{2}$ are $F$-terms that satisfy the expected $L>1$ non-renormalization properties, at least up to $L=5$.

The next term that arises in the low energy expansion is the dimension ten operator $s t_{8} \operatorname{tr}\left(F^{4}\right)$, which can be expressed as a term in the effective action given by a superspace integral, of the form $I_{1 / 4}=\int d^{10} x \int d^{16} \theta \theta^{8} \operatorname{tr}\left(W^{4}\right)$, where $W_{\alpha}$ is the gaugino superfield ( $\alpha$ is a space-time spinor index). This expression is schematic since we have not specified how to factor out the eight powers of $\theta$ in a covariant manner. However, this term is a "fake" $F$-term because it can be rewritten as a $D$-term, at least after compactification to dimension $D<10$. For example, in four dimensions it has the form $I_{1 / 4}=\int d^{4} x \int d^{16} \theta K$, where $K=\operatorname{tr}\left(\varphi^{i} \varphi_{i}\right)$ is the Konishi operator and $\varphi_{i}$ is the scalar superfield in the 6 of the R-symmetry group $\mathrm{SO}(6) \sim \mathrm{SU}(4)[6,26]$. The fact that this integral contains $\partial^{2} t_{8} \operatorname{tr}\left(F^{4}\right)$ follows from the nonlinear completion of the linearised $\mathcal{N}=4$ superfield. Our string calculations will confirm that $\partial^{2} t_{8} \operatorname{tr}\left(F^{4}\right)$ is not protected against quantum corrections and receives contributions for all $L>1$, as expected for $D$-terms. We will also show from string theory that the corresponding double-trace contribution $s t_{8}\left(\operatorname{tr} F^{2}\right)^{2} \sim \partial^{2} t_{8}\left(\operatorname{tr} F^{2}\right)^{2}$ only receives perturbative corrections up to two loops $(L \leq 2)$, as expected for a protected $F$-term that cannot be written as a $D$-term.

Furthermore, the dimension-twelve double-trace operator $s^{2} t_{8}\left(\operatorname{tr} F^{2}\right)^{2}$ can be expressed as a term in the effective action, $I_{1 / 8}=\int d^{4} x \int d^{16} \theta \theta^{4}\left(\operatorname{tr} W^{2}\right)^{2}$, which can also be rewritten as a $D$-term using $\int d^{4} x \int d^{16} \theta K^{2}$ and $\int d^{4} x \int d^{16} \theta T^{2}$ where $T_{A B}$ is the symmetric traceless supercurrent [26]. An analogous description of this $D$-term in terms of a scalar superfield should exist in all dimensions with $D<10$. We will again use the string theory multiloop amplitude prescription to argue that this double-trace contribution $s^{2} t_{8}\left(\operatorname{tr} F^{2}\right)^{2} \sim$ $\partial^{4} t_{8}\left(\operatorname{tr} F^{2}\right)^{2}$ receives perturbative corrections at all loop orders, as expected for a $D$-term.

\subsection{Organization of the paper}

In section 2 we will review the construction of scattering amplitudes on orientable planar world-sheets with $L$ open-string loops, in the non-minimal version of the pure spinor formalism. This describes open strings moving in $D$ dimensions and scattering on $N$ coincident $D p$-branes (where $D=p+1$ ). The amplitude for the scattering of four gluons will be considered in section 3 , where we will highlight the distinction between contribu- 
tions from single trace and double trace terms. We will also make a short comment that supports the arguments concerning $F$-terms in graviton scattering in type II closed string theories in [3]. In section 4 we will discuss the contributions of world-sheet handles, which are associated with the coupling of the closed-string (i.e., gravitational) sector and generate contributions suppressed by $O\left(1 / N^{2}\right)$ relative to the zero-handle terms. In section 5 we will show how the structure of these string theory expressions explains the pattern of ultraviolet divergences in maximally supersymmetric $\mathrm{U}(N)$ Yang-Mills theory at $L$ loops in $D$ dimensions. Whereas the onset of ultraviolet divergences of the single-trace term $s t_{8} \operatorname{tr}\left(F^{4}\right)$ to the $L$-loop amplitude occurs in dimensions $D=4+6 / L$, our results imply that the dimensional dependence of the double-trace terms is different. Given the overall prefactors described above, together with dimensional analysis we will see that ultraviolet divergences for the double-trace terms arise when:

- $D=8$ for the $L=1$ term $t_{8}\left(\operatorname{tr} F^{2}\right)^{2}$;

- $D=7$ for the $L=2$ term $s t_{8}\left(\operatorname{tr} F^{2}\right)^{2}$;

- $D=4+8 / L$ for the term $s^{2} t_{8}\left(\operatorname{tr} F^{2}\right)^{2}$ at $L \geq 3$.

This explains the apparent puzzles that have arisen in the explicit multi-loop calculations, where the double trace $t_{8}\left(\operatorname{tr} F^{2}\right)^{2}$ contribution to the $L$-loop counter-term is absent in dimensions $D=4+6 / L$ for $L=3$ and $L=4$ (as reviewed in [24]). Finally, we will summarize our results in section 6 and make some preliminary comments concerning higherpoint gluon amplitudes.

\section{Open-string scattering amplitudes in the pure spinor formalism}

The functional integral that defines the scattering amplitude with $M$ external massless ground states ("gluons") includes a sum over boundaries, handles and cross-caps (for theories with non-orientable world-sheets). Recall that a world-sheet with $M$ open-string vertices and with $B$ boundaries, $H$ handles and $C$ cross-caps is weighted with a factor $g_{s}^{-\chi}$, where $\chi$, the Euler number, is given by $\chi=2-2 H-B-C$ and where $g_{s}$ is the string coupling. When describing the scattering of open strings on a collection of $D p$-branes we need to consider orientable world-sheets, so that $C=0$. In addition we will initially neglect the contributions of world-sheets with handles so we will set $H=0$. We are interested in taking the limit in which gravity decouples from Yang-Mills and handles describe the gravitational contributions. However, handles also contribute finite residual pieces to pure super-Yang-Mills amplitudes. These contributions are suppressed by at least two powers of $N$ in the large $N$ limit (since one handle takes the place of two free boundaries). So we will be interested initially in the $L=B-1$ - loop oriented open string corrections with $\chi=1-L$. In the low-energy limit, these open superstring amplitudes describe $\mathrm{U}(N)$ super-Yang-Mills amplitudes to leading order in $1 / N^{2}$.

A general property of these open-string amplitudes is the possibility of divergences associated with closed strings coupling to the $D p$-brane via the world-sheet boundaries. The simplest example is the single-trace contribution at one loop, $L=1(B=2)$, which may 
be viewed as a cylinder carrying zero $(p+1)$-dimensional longitudinal momentum and with both boundaries fixed at the same transverse point. The potential divergence arises from the massless state propagating in the cylinder, which gives a contribution proportional to

$$
\lim _{x \rightarrow 0} \int^{x^{-1}} d^{9-p} q^{\perp}\left(q^{\perp}\right)^{-2}
$$

where $q^{\perp}$ is the transverse momentum in the cylinder, which is integrated in order to fix the boundaries at the same transverse positions. This expression diverges for $p \geq 7$, which indicates that the gravitational back reactions of $D p$-branes with $p \geq 7$ cannot be neglected. In the following, we will restrict our considerations to the situation in which these gravitational effects can be ignored, so we will be considering Yang-Mills in $D<8$ dimensions.

\subsection{The multi-loop functional integral for world sheets with $B$ boundaries}

The $M$-gluon amplitude is expressed as a sum of terms in which the $M$ vertex operators are partitioned among the $B$ boundaries in all possible ways and there is a sum over the order of the operators attached to each boundary. This gives

$$
A_{L}=\sum_{\text {orderings }} g_{s}^{B-2} \operatorname{tr}\left(T_{a_{1}^{(1)}} \cdots T_{a_{n_{1}}^{(1)}}\right) \cdots \operatorname{tr}\left(T_{a_{1}^{(B)}} \cdots T_{a_{n_{B}}^{(B)}}\right) \mathcal{A}_{L}^{\left(a_{1}^{(1)} \cdots a_{n_{1}}^{(1)}\right) \cdots\left(a_{1}^{(B)} \cdots a_{n_{B}}^{(B)}\right)}
$$

where $\left\{n_{r}\right\}$ is the number of vertex operators attached to each boundary labelled $r=$ $1, \ldots, B$ and $\sum_{r=1}^{B} n_{r}=M$. The quantity $\mathcal{A}_{L}^{\left(a_{1}^{(1)} \cdots a_{n_{1}}^{(1)}\right) \cdots\left(a_{1}^{(B)} \cdots a_{n_{B}}^{(B)}\right)}$ is the colour-ordered partial amplitude. The sum is over all partitions of the $M$ vertex operators on the $B$ boundaries, including all possible orderings of the operators on each boundary. The gauge group generators, $T_{a_{i}}$ are $N \times N$ matrices with indices in the defining representation of the gauge group, $\mathrm{U}(N)$ (which is the Chan-Paton prescription). Such a surface has $3 B=3 L+3$ real moduli for $L>1$ (and one modulus for $L=1$ ). Note that there is a factor of $\operatorname{tr}(1)=N$ for each boundary that has no vertex operators attached, leading to an overall factor of $N^{B_{f}}$ (where $B_{f}$ is the number of free boundaries in a given term in the sum).

In the non minimal pure spinor formalism the prescription for each colour-ordered open-string amplitude is given (for $B=L+1>2$ ) by

$$
\mathcal{A}_{L}^{\left(a_{1}^{(1)} \cdots a_{n_{1}}^{(1)}\right) \cdots\left(a_{1}^{(B)} \cdots a_{n_{B}}^{(B)}\right)}=\int d^{3 B-6} \tau\left\langle\prod_{i=1}^{3 B-6}\left(\mu_{i} \mid b\right) \mathcal{N} \prod_{i=1}^{M} \int d t_{i}: \mathcal{V}_{i}^{a_{i}}\left(t_{i}\right) e^{i k^{i} \cdot x}:\right\rangle
$$

where $(\mu \mid b):=\int d^{2} y \mu_{\bar{z}}^{z} b_{z z}$ (and $\mu_{\bar{z}}^{z}\left(\tau_{a}\right)=g_{z \bar{z}} \partial g^{z z} / \partial \tau_{a}$ is the Beltrami differential) and $\tau_{a}$ are the Teichmüller parameters of the bordered Riemann surface. For a given colour factor the positions of the vertex operators $\mathcal{V}_{i}^{a_{i}}$ are integrated in a given order along the boundary.

The angular bracket $\langle\cdots\rangle$ represents the path integral over the matter fields $\left[x^{m}, \theta^{\alpha}, p_{\alpha}\right]$ and the pure spinor ghosts which consist of left-movers, $\left[\lambda^{a}, w_{a}, \bar{\lambda}_{\alpha}, \bar{w}^{a}, r_{\alpha}, s^{\alpha}\right]$, and rightmovers, $\left[\tilde{\lambda}^{\alpha}, \tilde{w}_{\alpha}, \tilde{\bar{\lambda}}_{\alpha}, \tilde{\bar{w}}^{\alpha}, \tilde{r}_{\alpha}, \tilde{s}^{\alpha}\right]$, weighted by the pure spinor action

$$
\langle\cdots\rangle=\int \mathcal{D}^{10} x \mathcal{D}^{16} \theta \int[\mathcal{D} \lambda][\mathcal{D} \bar{\lambda}][\mathcal{D} r] \prod_{I=1}^{L} \int\left[\mathcal{D} w^{I}\right]\left[\mathcal{D} \bar{w}^{I}\right]\left[\mathcal{D} s^{I}\right] \cdots e^{-S_{p s}},
$$


where the action is (setting $2 \pi \alpha^{\prime}=1$ )

$$
\begin{gathered}
S_{p s}=\int d^{2} z\left(\frac{1}{2} \partial x^{m} \bar{\partial} x_{m}+p_{\alpha} \bar{\partial} \theta^{\alpha}+w_{\alpha} \bar{\partial} \lambda^{\alpha}+\bar{w}^{\alpha} \bar{\partial} \bar{\lambda}_{\alpha}+s^{\alpha} \bar{\partial} r_{\alpha}\right. \\
\left.+\tilde{p}_{\alpha} \tilde{\partial} \tilde{\theta}^{\alpha}+\tilde{w}_{\alpha} \tilde{\partial} \tilde{\lambda}^{\alpha}+\tilde{\bar{w}}^{\alpha} \tilde{\partial} \tilde{\bar{\lambda}}_{\alpha}+\tilde{s}^{\alpha} \tilde{\partial} \tilde{r}_{\alpha}\right) .
\end{gathered}
$$

This integral generically needs to be regularised by introducing the quantity $\mathcal{N}$ that will be reviewed below.

The pure spinor ghosts $\lambda^{\alpha}, \bar{\lambda}_{\alpha}$ and $r_{\alpha}$ satisfy the constraints

$$
\lambda \gamma^{m} \lambda=0, \quad \bar{\lambda} \gamma^{m} \bar{\lambda}=0, \quad \bar{\lambda} \gamma^{m} r=0,
$$

which implies that they each have eleven independent components. A conformal weight zero field has a single zero mode so $\theta^{\alpha}$ has 16 fermionic zero modes and $r_{\alpha}$ has 11 fermionic zero modes, which all have to be saturated in the functional integral. Likewise, each conformal weight one field has $L$ real zero modes. This means that $p_{\alpha}$ has $16 L$ real zero modes while $s^{\alpha}$ has $11 L$, which also need to be saturated. The bosonic pure spinor ghosts $\lambda^{\alpha}$ and $\bar{\lambda}_{\alpha}$ each have 11 independent components which need to be integrated. Singularities in these integrals need to be regulated $[1,2,27,28]$. Similarly, the conjugate bosonic variables, $w_{\alpha}$ and $\bar{w}^{\alpha}$, each have $11 L$ components.

The integration measures are given by

$$
\begin{aligned}
\lambda^{\alpha} \lambda^{\beta} \lambda^{\gamma}[\mathcal{D} \lambda] & =\left(\epsilon \mathcal{T}^{-1}\right)_{k_{1} \cdots k_{11}}^{\alpha \beta \gamma} \mathcal{D} \lambda^{k_{1}} \cdots \mathcal{D} \lambda^{k_{11}} \\
{[\mathcal{D} \bar{\lambda}][\mathcal{D} r] } & =\mathcal{D} \bar{\lambda}_{\alpha_{1}} \wedge \cdots \wedge \mathcal{D} \bar{\lambda}_{\alpha_{11}} \times \partial_{r_{\alpha_{1}}} \wedge \cdots \wedge \partial_{r_{\alpha_{11}}}
\end{aligned}
$$

where the tensor $\mathcal{T}$, which is totally antisymmetric on the $k_{i}$ indices and fully symmetric and $\gamma$-traceless on the $\alpha \beta \gamma$ indices, has the form [1]

$$
(\epsilon \mathcal{T})_{\alpha \beta \gamma}^{k_{1} \cdots k_{11}}=\epsilon_{16}^{k_{1} \cdots k_{11} r_{1} \cdots r_{5}}\left(\gamma^{m}\right)_{\left(\left(\alpha\left|r_{1}\right|\right.\right.}\left(\gamma^{n}\right)_{\beta\left|r_{2}\right|}\left(\gamma^{p}\right)_{\gamma)) r_{3}}\left(\gamma_{m n p}\right)_{r_{4} r_{5}} .
$$

The integration measure of the conjugate ghosts is given by

$$
\begin{aligned}
\lambda^{\alpha_{1}} \cdots \lambda^{\alpha_{8}}\left[\mathcal{D} w^{I}\right] & =M_{m_{1} n_{1} \cdots m_{10} n_{10}}^{\alpha_{1} \cdots \alpha_{8}} \mathcal{D} N^{m_{1} n_{1} I} \cdots \mathcal{D} N^{m_{10} n_{10} I} \mathcal{D} J^{I} \\
{\left[\mathcal{D} \bar{w}^{I}\right]\left[\mathcal{D} s^{I}\right] } & =\prod_{i=1}^{10} \mathcal{D} \bar{N}_{m_{i} n_{i}}^{I} \wedge \mathcal{D} \bar{J}^{I} \wedge \prod_{i=1}^{10} \partial_{S_{m_{i} n_{i}}^{I}} \wedge \partial_{S^{I}}
\end{aligned}
$$

where

$$
\begin{aligned}
M_{m_{1} n_{1} \cdots m_{10} n_{10}}^{\alpha_{1} \cdots \alpha_{8}}= & \left(\gamma_{m_{1} n_{1} m_{2} m_{3} m_{4}}\right)^{\left(\left(\alpha_{1} \alpha_{2}\right.\right.}\left(\gamma_{m_{5} n_{5} n_{2} m_{6} m_{7}}\right)^{\alpha_{3} \alpha_{4}} \\
& \left(\gamma_{m_{8} n_{8} n_{3} n_{6} m_{9}}\right)^{\alpha_{5} \alpha_{6}}\left(\gamma_{m_{10} n_{10} n_{4} n_{7} n_{9}}\right)^{\left.\left.\alpha_{7} \alpha_{8}\right)\right)}
\end{aligned}
$$

and $((\cdots))$ means that one considers the symmetrised $\gamma$-traceless part. The quantities

$$
\begin{aligned}
N^{m n} & =\lambda \gamma^{m n} w, & J & =\lambda^{\alpha} w_{\alpha} \\
\bar{N}^{m n} & =\bar{\lambda} \gamma^{m n} \bar{w}-r \gamma^{m n} s, & \bar{J} & =\bar{\lambda} \cdot \bar{w}-r \cdot s \quad S^{m n}=\bar{\lambda} \gamma^{m n} s, \quad S=\bar{\lambda} \cdot s,
\end{aligned}
$$

are conserved world-sheet currents. 
The $b$-ghost is a composite quantity, which is defined to satisfy the BRST condition $[Q, b]=T$, where $Q$ is the BRST charge and $T$ is the energy-momentum tensor. This takes the form $[1,2]$,

$$
b=s \partial \bar{\lambda}+\frac{\bar{\lambda}_{\alpha} \mathbf{b}^{\alpha}}{\lambda \cdot \bar{\lambda}}
$$

and

$$
\mathbf{b}^{\alpha} \equiv G^{\alpha}+\frac{r_{\beta}}{\lambda \cdot \bar{\lambda}} H^{\alpha \beta}+\frac{r_{\beta} r_{\gamma}}{(\lambda \cdot \bar{\lambda})^{2}} K^{\alpha \beta \gamma}+\frac{r_{\beta} r_{\gamma} r_{\delta}}{(\lambda \cdot \bar{\lambda})^{3}} L^{\alpha \beta \gamma \delta}
$$

where

$$
\begin{aligned}
G^{\alpha} & \equiv \frac{1}{2} \Pi^{m}\left(\gamma_{m} d\right)^{\alpha}-\frac{1}{4} N_{m n}\left(\gamma^{m n} \partial \theta\right)^{\alpha}-\frac{1}{4} J \partial \theta^{\alpha}-\frac{1}{4} \partial^{2} \theta^{\alpha} \\
H^{\alpha \beta} & \equiv \frac{1}{192}\left(\gamma^{m n p}\right)^{\alpha \beta}\left(\left(d \gamma_{m n p} d\right)+4 ! N_{m n} \Pi_{p}\right) \\
K^{\alpha \beta \gamma} & \equiv \frac{1}{16}\left(\gamma_{m n p}\right)^{[\alpha \beta}\left(\gamma^{m} d\right)^{\gamma]} N^{n p} \\
L^{\alpha \beta \gamma \delta} & =\frac{1}{128}\left(\gamma_{m n p}\right)^{[\alpha \beta}\left(\gamma^{p q r}\right)^{\gamma \delta]} N^{n m} N_{q r} .
\end{aligned}
$$

The pieces of the $b$-ghost satisfy the relations

$$
\begin{aligned}
\left\{Q, G^{\alpha}\right\} & =\lambda^{\alpha} T, & \left\{Q, H^{\alpha \beta}\right\} & =\lambda^{\alpha} G^{\beta}, \\
\left\{Q, K^{\alpha \beta \gamma}\right\} & =\lambda^{\alpha} H^{\beta \gamma}, & \left\{Q, L^{\alpha \beta \gamma \delta}\right\} & =\lambda^{\alpha} H^{\beta \gamma \delta} .
\end{aligned}
$$

The regulator $\mathcal{N}$ in $(2.3)$ given by

$$
\begin{aligned}
\mathcal{N}= & \exp [Q, \Psi] \\
= & \exp [-\lambda \cdot \bar{\lambda}-r \cdot \theta] \\
& \times \exp \left[-\sum_{I=1}^{L}\left(\frac{1}{2} N_{m n}^{I} \bar{N}^{I m n}+J_{I} \bar{J}^{I}\right)\right] \\
& \times \exp \left[-\sum_{I=1}^{L} \frac{1}{4} S_{m n}^{I}\left(d^{I} \gamma^{m n} \lambda\right)+S^{I}\left(\lambda d^{I}\right)\right],
\end{aligned}
$$

In this expression we have defined the $I^{\prime}$ 'th zero mode of any current in $(2.11), \mathcal{O}^{I}$, as the integral of $\mathcal{O}$ around the $I^{\prime}$ th $a$-cycle of the doubled open-string world-sheet, $\mathcal{O}^{I}=\oint_{a_{I}} d z \mathcal{O}$.

\subsection{The open-string vertex operator}

An open string vertex operator in (2.3) attached to a point $t$ on a boundary, $\mathcal{V}^{a}(t) e^{i k \cdot x}$, is the $k^{\prime}$ th Fourier mode of the position-space superfield given by

$$
\mathcal{V}^{a}(x, \theta)=A_{\alpha}^{a}(x, \theta) \partial \theta^{\alpha}+A_{m}^{a}(x, \theta) \Pi^{m}+W^{a \alpha}(x, \theta) d_{\alpha}+\frac{1}{2} N^{m n} \mathcal{F}_{m n}^{a}(x, \theta),
$$

where $\Pi^{m}=\partial x^{m}+i / 2\left(\theta \gamma^{m} \partial \theta\right), d_{\alpha}=p_{\alpha}-i / 2\left(\theta \gamma_{m}\right)_{\alpha}\left(\partial x^{m}+\left(\theta \gamma^{m} \partial \theta\right) / 4\right)$, and $A_{\alpha}^{a}, A_{m}^{a}$, $W^{a \alpha}$ and $\mathcal{F}_{m n}^{a}$ are the $\mathcal{N}=1 D=10$ super-Yang-Mills superfields,

$$
A_{\alpha}^{a}(x, \theta)=\frac{1}{2}\left(\gamma^{m} \theta\right)_{\alpha} a_{m}^{a}(x)-\frac{1}{3}\left(\chi^{a}(x) \gamma_{m} \theta\right)\left(\gamma^{m} \theta\right)_{\alpha}-\frac{1}{32} F_{m n}^{a}(x)\left(\gamma_{p} \theta\right)_{\alpha}\left(\theta \gamma^{m n p} \theta\right)+\cdots,
$$


$a_{m}^{a}(x)$ and $\chi^{\alpha a}(x)$ are the gluon and gluino fields and $F_{m n}^{a}=\partial_{[m} a_{n]}^{a}$, and

$$
\begin{aligned}
\left(\gamma^{m}\right)_{\alpha \beta} A_{m} & =D_{\alpha} A_{\beta}+D_{\beta} A_{\alpha} \\
\left(\gamma_{m}\right)_{\alpha \beta} W^{\beta} & =D_{\alpha} A_{m}-\partial_{m} A_{\alpha} \\
D_{\alpha} W^{\beta} & =\frac{1}{4}\left(\gamma^{m n}\right)_{\alpha}{ }^{\beta} \mathcal{F}_{m n},
\end{aligned}
$$

where $D_{\alpha}=\partial / \partial \theta_{\alpha}+1 / 2\left(\theta \gamma^{m}\right)_{\alpha} \partial_{m}$ is the supersymmetric derivative. The superfield $W^{\alpha}(x, \theta)$ takes the form

$W^{a \alpha}=\chi^{a \alpha}-\frac{1}{4}\left(\gamma^{m n} \theta\right)^{\alpha} F_{m n}^{a}+\frac{1}{4}\left(\gamma^{m n} \theta\right)\left(\partial_{m} \chi^{a} \gamma^{m} \theta\right)+\frac{1}{2 \cdot 4 !}\left(\gamma^{p q} \theta\right)^{\alpha}\left(\theta \gamma_{q} \gamma^{m n} \theta\right) \partial_{p} F_{m n}^{a}+O\left(\theta^{4}\right)$.

The first two terms in (2.17) can be expressed by means of a normal coordinate expansion around $Z_{0}=\left(x_{0}^{m}, \theta_{0}^{\alpha}\right)$, giving

$$
d Z^{M} A_{M}=A_{M}\left(Z_{0}\right) d Z^{M}+\mathcal{F}_{M N}\left(Z-Z_{0}\right)^{M} d Z^{N}+\cdots
$$

The first term in this expression can be ignored since it can be written as the surface term $d\left(A_{M}\left(Z_{0}\right) Z^{M}\right)$ which decouples using the standard canceled propagator argument. And because $Z-Z_{0}$ does not contain $\theta$ zero modes, $\mathcal{F}_{M N}\left(Z-Z_{0}\right)^{M} d Z^{N}+\cdots$ only contributes to terms in the effective action which are higher order in derivatives than the terms coming from $d_{\alpha} W^{\alpha}$. This is easy to see since $d_{\alpha} W^{\alpha}$ can contribute a $d_{\alpha}$ zero mode whereas the term $W^{\alpha}\left[\left(\theta-\theta_{0}\right) d X-\left(X-X_{0}\right) d \theta\right]_{\alpha}$ in $\mathcal{F}_{M N}\left(Z-Z_{0}\right)^{M} d Z^{N}$ cannot contribute either $d_{\alpha}$ or $\theta^{\alpha}$ zero modes. Furthermore, the term $N^{m n} \mathcal{F}_{m n}$ in (2.17) does not contain $d_{\alpha}$ zero modes so it also only affects terms which are higher order in derivatives than terms coming from $W^{\alpha} d_{\alpha}$. Therefore, when analyzing the terms in the effective action of lowest dimension at a given genus, one only needs to consider the contribution from $W^{\alpha} d_{\alpha}$ in (2.17).

\section{The scattering of four open strings}

We will now specialise to the scattering of four massless open-string ground state gluons with momenta $k_{r}(r=1,2,3,4)$ satisfying $k_{r}^{2}=0$. In this case there are two distinct contributions at each order in perturbation theory, which differ in the way their gauge indices are contracted. One of these is the single trace term in which all vertex operators are attached to one boundary, resulting in an overall factor of $N^{L} s^{\gamma_{L}} t_{8} \operatorname{tr}\left(F^{4}\right)$ in the low energy limit of the amplitude without handles $H=0$. For $L=1$ we know $\gamma_{0}=\gamma_{1}=0$ and we will argue shortly that $\gamma_{L}=1$ for $L>1$. As we will see later in this section, $s t_{8} \operatorname{tr}\left(F^{4}\right)$ gets contributions from all values of $L>1$, as expected for a $D$-term. The second kind of contribution is the double trace term, arising when the four vertex operators are partitioned in pairs between two boundaries, resulting in a factor $N^{L-1} s^{\beta_{L}} t_{8}\left(\operatorname{tr} F^{2}\right)^{2}$. There is no such contribution at tree level $(L=0)$ and we know that $\beta_{1}=0$. We will see shortly that $\beta_{L}=$ $\lceil L / 2\rceil$ for $1<L \leq 4$ (recalling that $\lceil x\rceil$ denotes the smallest integer greater or equal than $x)$ again up to at least $L=4$. Terms of this form will turn out to be " $F$-terms" when $L \leq 2$.

The powers of momenta, $2 \gamma_{L}$ and $2 \beta_{L}$ in the low energy amplitude depend crucially on the analysis of the integrations over fermionic zero modes. 


\subsection{Zero-mode integrals and momentum prefactors}

We will now discuss the integration over the fermionic zero modes that need to be saturated in order to obtain a non-zero contribution to the amplitude. As explained in $[2,3]$, the regulator of (2.16) regularizes divergences in the pure spinor functional integral coming from $\lambda, \bar{\lambda} \rightarrow \infty$ and in the process provides essential fermionic zero modes. However, to regularise potential divergences from the $\lambda, \bar{\lambda} \rightarrow 0$ endpoint, one needs to introduce a small $\lambda$ regulator which is more complicated because it involves non-zero modes of the worldsheet fields. Fortunately, it was shown in $[2,3]$ that this more complicated regulator is unnecessary for evaluating contributions to " $F$-terms" in the effective action. Here, we are defining " $F$-terms" as any term in the effective action where the external vertex operators contribute fewer than sixteen $\theta$ zero modes. In other words, at least one $\theta$ zero mode must come from the regulator of $(2.16)$ when evaluating an " $F$-term".

To absorb the $16 L$ zero modes of $d$ in the most efficient manner in an $L$-loop amplitude, the $(3 L-3) b$-ghosts should contribute the terms $(\bar{\lambda} \Pi d)^{L-2}\left(\bar{\lambda} r d^{2}\right)^{2 L-1}$. Note that increasing the relative number of $\left(\bar{\lambda} r d^{2}\right)$ terms will allow some $d$ 's to contribute nonzero modes. But since each such term includes an extra $r$, it will increase the number of $\theta$ zero modes which come from the external vertex operators. So changing a $d_{\alpha}$ and $\theta^{\beta}$ zero mode to a $d_{\alpha}$ and $\theta^{\beta}$ nonzero mode forces the external vertex operators to contribute two extra $\theta$ zero modes, which is equivalent to adding a factor of momentum. So each contraction of $d_{\alpha}$ with $\theta^{\beta}$ in the computation adds a factor of momentum to the term in the effective action.

We now wish to isolate the terms of lowest dimension - in other words, the terms with the least number of $\theta$ 's taken out of the vertex operators. Using the normal coordinate expansion of (2.21) it is easy to see that the vertex operator of lowest dimension is the superfield $W^{\alpha}$. Using the above contribution from the $b$-ghosts, the term with the lowest power of momentum in the $L$-loop amplitude (for $L>1$ ) is proportional to the correlation function

$$
\int d^{3(L-1)} \tau\left\langle(r \cdot \theta)^{12-2 L}(S \lambda d)^{11 L}(\bar{\lambda} \Pi d)^{L-2}\left(\bar{\lambda} r d^{2}\right)^{2 L-1}(\lambda \bar{\lambda})^{-5 L+4}(W d)^{4}\right\rangle .
$$

In this expression the first two factors come from expanding the regulator $\mathcal{N}$, the subsequent four factors come from the $3 L-3$ powers of the $b$-ghost, and the last factor comes from the vertex operators. We see that there are $12-2 L$ powers of $\theta$ and therefore the term of lowest dimension at $L$ loops is proportional to

$$
\int d^{16} \theta \theta^{12-2 L} W^{4} \sim \partial^{L} t_{8} F^{4} .
$$

This expression is symbolic since we have not specified the way in which the gauge indices are contracted or the details of how the derivatives act on the four fields, but these are determined by the explicit calculations. Since only terms with an even number of momenta can be non-vanishing, one finds that $\partial^{2} t_{8} F^{4}$ is the term of lowest dimension at $L=2, \partial^{4} t_{8} F^{4}$ is the term of lowest dimension at $L=3$ and $L=4$, and $\partial^{6} t_{8} F^{4}$ is the term of lowest dimension for $L \geq 5$.

This expression suggests that the $D$-term of lowest dimension is $\int d^{16} \theta W^{4} \sim \partial^{6} F^{4}$. However, this is too naive since it assumes that the remaining integrations over the non-zero 
modes in (3.1) do not contribute inverse derivative factors such as $\left(k_{r} \cdot k_{s}\right)^{-1}$. Such factors do arise and play an important rôle in determining which terms are genuine $F$-terms and which are fake $F$-terms. The systematics of these inverse derivatives is the subject of the next subsection.

We will also consider the extension of this argument to four-graviton amplitudes in the type II closed string theory where it was argued in [3] that terms proportional to $\partial^{2 k} R^{4}$ with $k \leq 5$ are $F$-terms that do not receive corrections beyond $k$ loops. This also assumed the absence of inverse powers of momentum arising from non-zero modes, which we will justify at the end of the next subsection for terms that do not require the complicated small $\lambda$ regulator.

\subsection{Inverse derivative factors}

When computing a massless four-point $L$-loop amplitude, one expects to get inverse derivative factors of $\left(k_{1} \cdot k_{2}\right)^{-1}$ coming from massless poles when $\left(k_{1}+k_{2}\right)^{2}=2 k_{1} \cdot k_{2}=0$. But the on-shell massless three-point amplitude vanishes beyond tree level (i.e., for $L \geq 1$ ) in superstring theory, so the massless four-point loop amplitude cannot have a physical pole when $k_{1} \cdot k_{2}=0$. Nevertheless, there is the possibility that inverse factors of $\left(k_{1} \cdot k_{2}\right)^{-1}$ could cancel factors of $\left(k_{1} \cdot k_{2}\right)$ in the prefactor of the amplitude from the zero mode saturation. As will now be discussed, these inverse factors can come from performing the contractions over the non-zero modes of the world-sheet fields and integrating over the moduli of the amplitude. This could in principle reduce the $D$-term of lowest dimension from $\int d^{16} \theta W^{4}=\partial^{6} F^{4}$ to a term with fewer derivatives.

In the superstring computation, these inverse derivative factors arise from the boundary of moduli space where either two vertex operators collide or where the string world-sheet splits into two world-sheets connected by a long open string strip (or closed string tube). For example, a factor of $\left(k_{1} \cdot k_{2}\right)^{-1}$ could arise from the region of the integral $\int d z_{2} V_{2}\left(z_{2}\right) V_{1}\left(z_{1}\right)$ when $z_{2}$ approaches $z_{1}$. This inverse derivative factor occurs if $V_{2}\left(z_{2}\right) V_{1}\left(z_{1}\right)$ has a term in its OPE which goes like $\left(z_{2}-z_{1}\right)^{-1+k_{1} \cdot k_{2}}$ so that $\int d z_{2} V_{2}\left(z_{2}\right) V_{1}\left(z_{1}\right) \sim \int d z z^{-1+k_{1} \cdot k_{2}} \sim$ $\left(k_{1} \cdot k_{2}\right)^{-1}$. Similarly, a factor of $\left(k_{1} \cdot k_{2}\right)^{-1}$ could arise from the limit in which the $L$-loop world-sheet degenerates into $L_{1}$-loop and $L_{2}$-loop world-sheets connected by a long open string strip (or closed string tube) where $L=L_{1}+L_{2}$ and $k_{1}+k_{2}$ is the momentum going through the strip (or tube). Such an inverse derivative factor could come from the $y \rightarrow 0$ region of the integral $\int d y y^{-1+k_{1} \cdot k_{2}} \sim\left(k_{1} \cdot k_{2}\right)^{-1}$ where $-\log (y)$ is the length of the open string strip, or from the $y \rightarrow 0$ region of the integral $\int d^{2} y|y|^{-2+k_{1} \cdot k_{2}} \sim\left(k_{1} \cdot k_{2}\right)^{-1}$ where $-\log (y)$ is the complex modulus of the closed string tube.

It will now be shown that when $L<5$, the only possible source of inverse derivative factors comes from the collision of vertex operators. Furthermore, these inverse derivative factors only affect the low-energy dependence of the $s^{\gamma_{L}} t_{8} \operatorname{tr}\left(F^{4}\right)$ term, and do not affect the $s^{\beta_{L}} t_{8}\left(\operatorname{tr} F^{2}\right)^{2}$ term. For $L \geq 5$, one can also get inverse derivative factors from the degeneration of the surface, and these inverse factors affect both the $s^{\gamma_{L}} t_{8} \operatorname{tr}\left(F^{4}\right)$ and $s^{\beta_{L}} t_{8}\left(\operatorname{tr} F^{2}\right)^{2}$ terms. It will also be shown that neither of these two sources of inverse derivative factors affect the low-energy dependence of the $s^{k} R^{4}$ terms in closed superstring scattering (at least for terms which do not involve the small $\lambda$ regulator). 
We shall first discuss possible inverse derivative factors coming from the collision of vertex operators. As explained in subsection 3.1, the term of fewest derivatives in the effective action comes if each of the four vertex operators contributes $W^{\alpha} d_{\alpha}$ and these four $d_{\alpha}$ 's only contribute zero modes. When two such vertex operators collide, e.g. $V_{1}\left(z_{1}\right)$ and $V_{2}\left(z_{2}\right)$, the resulting OPE is simply $W_{1}^{\alpha} d_{\alpha} W_{2}^{\beta} d_{\beta}\left(z_{2}-z_{1}\right)^{k_{1} \cdot k_{2}}$. But to get an inverse derivative factor of $\left(k_{1} \cdot k_{2}\right)^{-1}$, the OPE must have a term proportional to $\left(z_{2}-z_{1}\right)^{-1+k_{1} \cdot k_{2}}$. To get this additional factor of $\left(z_{2}-z_{1}\right)^{-1}$, the $d_{\alpha}$ variable in one of the vertex operators must contribute a nonzero mode which contracts with a $\theta^{\alpha}$ variable in the other vertex operator.

Note that switching the order of the two vertex operators will reverse the sign from $\left(z_{2}-z_{1}\right)^{-1}$ to $\left(z_{1}-z_{2}\right)^{-1}$, so the resulting OPE is antisymmetric under exchange of the group theory factors $T_{1}$ and $T_{2}$. This immediately implies that this type of inverse derivative factor, which comes from colliding vertex operators, is not present for the $t_{8}\left(\operatorname{tr} F^{2}\right)^{2}$ term. If $V_{1}$ and $V_{2}$ are on the same boundary for the double-trace term, $\operatorname{tr}\left(T_{1} T_{2}\right)=\operatorname{tr}\left(T_{2} T_{1}\right)$ implies that the antisymmetric part of the OPE does not contribute. This is related to the fact that the gluon vertex operator coming from the pole in the OPE of the two external vertex operators would be a U(1) gluon which decouples from non-abelian states.

For the $t_{8} \operatorname{tr}\left(F^{4}\right)$ term, these colliding vertex operators could potentially reduce the number of derivatives. However, for this to happen, the missing $d_{\alpha}$ zero mode from the colliding vertex operator needs to be replaced by an extra $d_{\alpha}$ zero mode coming from the $b$ ghosts. The lowest value of $L$ for which this is possible is $L=3$, as can be seen from (3.1) - by changing the $(\bar{\lambda} \Pi d)$ contribution to a $\left(\bar{\lambda} r d^{2}\right)$ contribution, one gets an extra $d_{\alpha}$ zero mode. However, because one also gets an extra $r_{\alpha}$ zero mode and because one of the $\theta$ zero modes in the vertex operators was contracted with the $d_{\alpha}$ nonzero mode, the number of $\theta$ 's at $L=3$ coming from the vertex operators is increased from 10 to 12 . After including the inverse derivative factor of $\left(k_{1} \cdot k_{2}\right)^{-1}$, this means that the term with fewest derivatives at $L=3$ is

$$
\left(k_{1} \cdot k_{2}\right)^{-1}\left(\frac{\partial}{\partial \theta}\right)^{12} W^{4} \sim \partial^{2} t_{8} \operatorname{tr}\left(F^{4}\right) .
$$

If in addition to $V_{1}$ and $V_{2}$ colliding, one also had $V_{3}$ and $V_{4}$ colliding, one could potentially get an additional inverse derivative factor of $\left(k_{1} \cdot k_{2}\right)^{-2}$. In this case, one would need to get two extra $d_{\alpha}$ zero modes from the $b$ ghosts in order to replace the two $d_{\alpha}$ zero modes in the vertex operators which were contracted with $\theta$ 's. It is easy to see from (3.1) that this is possible at $L=4$ by changing two $(\bar{\lambda} \Pi d)$ contributions to $\left(\bar{\lambda} r d^{2}\right)$ contributions. This gives two extra $r_{\alpha}$ zero modes and, because two $\theta$ zero modes in the vertex operators are contracted, the number of $\theta$ 's at $L=4$ coming from the vertex operators is increased from 12 to 16. After including the inverse derivative factor of $\left(k_{1} \cdot k_{2}\right)^{-2}$, this means that the term with fewest derivatives at $L=4$ is

$$
\left(k_{1} \cdot k_{2}\right)^{-2}\left(\frac{\partial}{\partial \theta}\right)^{16} W^{4} \sim \partial^{2} t_{8} \operatorname{tr}\left(F^{4}\right),
$$

resulting in the same operator as in (3.3).

So we have seen that colliding vertex operators reduce the momentum dependence of the $t_{8} \operatorname{tr}\left(F^{4}\right)$ term from $\partial^{3} t_{8} \operatorname{tr}\left(F^{4}\right)$ to $\partial^{2} t_{8} \operatorname{tr}\left(F^{4}\right)$ at $L=3$, and from $\partial^{4} t_{8} \operatorname{tr}\left(F^{4}\right)$ to 
$\partial^{2} t_{8} \operatorname{tr}\left(F^{4}\right)$ at $L=4$. However, the momentum dependence of the double-trace term is unaffected and remains $\partial^{2[3 / 2\rceil} t_{8}\left(\operatorname{tr} F^{2}\right)^{2}=\partial^{4} t_{8}\left(\operatorname{tr} F^{2}\right)^{2}$ at $L=3$ and $\partial^{4} t_{8}\left(\operatorname{tr} F^{2}\right)^{2}$ at $L=4$.

We will now analyze the second possible source of inverse derivative factors coming from the degeneration of the $L=L_{1}+L_{2}$ world-sheet into two world-sheets with $L_{1}$ and $L_{2}$ loops. In practice we will find it convenient to describe the closed string version of this plumbing decomposition, which is related to the open string version by the usual doubling trick. So we will consider the degeneration of a genus $g=g_{1}+g_{2}$ closed-string world-sheet into two world-sheets of genus $g_{1}$ and $g_{2}$. To analyze this degeneration, it is convenient to use the standard "plumbing" decomposition of the surface into a genus $g_{1}$ surface with a small hole at $p_{1}$, a genus $g_{2}$ surface with a small hole at $p_{2}$, and a cylinder of length $-\log (y)$ connecting the two holes. The Beltrami differential for the cylinder length is $\int d y y^{-1}$, and the corresponding $b$ ghost is integrated around the circumference of the cylinder. The remaining $3 g-4 b$ ghosts are split into $3 g_{1}-2 b$ ghosts on the $g_{1}$ surface (with one of them at $p_{1}$ ) and $3 g_{2}-2 b$ ghosts on the $g_{2}$ surface (with one of them at $p_{2}$ ).

To compute the correlation function in this degeneration limit, it is convenient to map the cylinder of length $-\log (y)$ into an annulus which has one small boundary of radius $\sqrt{y}$ and one large boundary of radius $1 / \sqrt{y}$. The annulus coordinate will be called $w$, which is related to the cylinder coordinate $\rho$ by $w=e^{\rho}$. To get an inverse derivative factor, the correlation function on the annulus must contribute a factor of $y^{k_{1} \cdot k_{2}}$, so that after integrating over the moduli using the Beltrami differential for $y$, one gets a factor of $\int d y y^{-1} y^{k_{1} \cdot k_{2}} \sim\left(k_{1} \cdot k_{2}\right)^{-1}$.

The correlation function on the annulus is given by

$$
\left\langle V_{1}(w=\sqrt{y}) \int d w w b(w) V_{2}(w=\sqrt{1 / y})\right\rangle
$$

where $V_{1}$ comes from the operators on the genus $g_{1}$ surface, $V_{2}$ comes from the operators on the genus $g_{2}$ surface, and the $b$ ghost is integrated around the countour $|w|=1$. If gluons 1 and 2 are on the $g_{1}$ surface and gluons 3 and 4 are on the $g_{2}$ surface, $V_{1}$ will be proportional to $e^{i\left(k_{1}+k_{2}\right) x}$ and $V_{2}$ will be proportional to $e^{-i\left(k_{1}+k_{2}\right) x}$. So if the $b$ ghost does not contribute any $y$ dependence, the correlation function gives the desired factor of $y^{k_{1} \cdot k_{2}}$.

However, when $y \rightarrow 0$, the $b$ ghost cannot contribute any $d_{\alpha}$ or $\Pi$ zero modes. This is easy to see since when $y \rightarrow 0$, the $g$ holomorphic one-forms split into $g_{1}$ holomorphic one-forms which are non-vanishing only on the genus $g_{1}$ surface and $g_{2}$ holomorphic oneforms which are non-vanishing only on the genus $g_{2}$ surface. The $b$ ghost on the annulus contributes either the term $\bar{\lambda} \Pi d$ or $\bar{\lambda} r d^{2}$. In the first case, the nonzero mode of the $\Pi$ must contract with $e^{i\left(k_{1}+k_{2}\right) x}$ to give a factor of $k$, and the nonzero mode of $d_{\alpha}$ must contract with a $\theta^{\alpha}$ in $V_{1}$ or $V_{2}$. Furthermore, one needs an extra $d_{\alpha}$ zero mode to come from one of the other $b$ ghosts (which is possible when $L \geq 3$ as before). Putting all of these factors together, one gets a total factor of $k^{2}$ which cancels the inverse derivative factor of $\left(k_{1} \cdot k_{2}\right)^{-1}$. In the second case where the $b$ ghost on the annulus contributes $\bar{\lambda} r d^{2}$, the two nonzero modes of $d_{\alpha}$ must contract with two $\theta^{\alpha}$ 's in $V_{1}$ or $V_{2}$, and one needs two extra $d_{\alpha}$ zero modes to come from the other $b$ ghosts (which is possible when $L \geq 4$ ). Again putting these factors together, one gets a total factor of $k^{2}$ which cancels the inverse derivative factor of $\left(k_{1} \cdot k_{2}\right)^{-1}$. 
So degeneration of the surface cannot give inverse derivative factors when $L<5$. However, when $L \geq 5$, there are contributions to the low-energy effective action which require using the more complicated small $\lambda$ regulator. Although we will not go into details here, we will sketch how degeneration of the surface can give rise to inverse derivative factors when $L \geq 5$.

When $L=5$, there are $12 b$ ghosts which can contribute the term $\left(\bar{\lambda} r d^{2}\right)^{12} \sim r^{12} d^{24}$. This term diverges when $\lambda \rightarrow 0$ as $\lambda^{-12}$ which means one needs to use the complicated version of the regulator. Note that one of the $12 r$ 's in this term must contribute a nonzero mode (since there are only 11 independent $r_{\alpha}$ zero modes). As shown in $[2,27]$, the small $\lambda$ regulator involves a term $d_{\alpha} s^{\alpha}$ with nonzero modes, and after contracting the $s$ nonzero mode in the regulator with the $r_{\alpha}$ nonzero mode, one is left with a term proportional to $r^{11} d^{25}$. After including the $4 d_{\alpha}$ 's from the vertex operators and the $5 \times 11=55 d_{\alpha}$ zero modes from the regulator, one has a total of $84 d_{\alpha}$ 's. At $L=5$, one needs $80 d_{\alpha}$ zero modes, so 4 of the $84 d_{\alpha}$ 's can contribute nonzero modes.

If the $L=5$ surface degenerates in two places, one gets two inverse derivative factors which produce $\left(k_{1} \cdot k_{2}\right)^{-2}$. The two $b$ ghosts on the two annuli will involve these four $d_{\alpha}$ nonzero modes which will contract with four of the $\theta$ 's on $V_{1}$ or $V_{2}$. Since no $\theta$ 's come from the regulator, one needs to get $20 \theta$ 's from the vertex operators. So at $L=5$, the number of derivatives in the double-trace term is $k^{-4}\left(\frac{\partial}{\partial \theta}\right)^{20} \operatorname{tr}\left(W^{2}\right)^{2} \sim \partial^{4} t_{8}\left(\operatorname{tr} F^{2}\right)^{2}$. Note that this is one derivative lower than the $\partial^{5} t_{8}\left(\operatorname{tr} F^{2}\right)^{2}$ term one would get in the absence of inverse derivative factors.

For the single-trace term, one can use two of these four $d_{\alpha}$ nonzero modes to give an inverse derivative factor of $k^{-4}$ from colliding vertex operators $V_{1}$ with $V_{2}$ and $V_{3}$ with $V_{4}$. The remaining two $d_{\alpha}$ nonzero modes can be used in the $b$ ghost on an annulus which degenerates the $L=5$ surface in one place, which gives an additional factor of $k^{-2}$. Since each $d_{\alpha}$ nonzero mode is contracted with a $\theta$ from the vertex operators, one again needs $20 \theta$ 's from the vertex operators, so at $L=5$ the single-trace term is proportional to $k^{-6}\left(\frac{\partial}{\partial \theta}\right)^{20} \operatorname{tr}\left(W^{4}\right) \sim \partial^{2} t_{8} \operatorname{tr}\left(F^{4}\right)$.

So after including the inverse derivative factors coming from these two sources, one finds that the single-trace term is proportional to $\partial^{2} t_{8} \operatorname{tr}\left(F^{4}\right)$ for $L>1$ and the doubletrace term is proportional to $\partial^{2} t_{8}\left(\operatorname{tr} F^{2}\right)^{2}$ for $1<L \leq 2$ and $\partial^{4} t_{8}\left(\operatorname{tr} F^{2}\right)^{2}$ for $L>2$. This was verified up to $L=5$.

Finally, it is easy to show that for terms which do not involve the small $\lambda$ regulator, neither of these sources can contribute inverse derivative factors for closed string scattering. This is because the number of $d_{\alpha}$ and $\theta^{\alpha}$ nonzero modes is doubled (since one has both left and right-moving contributions), but the number of inverse derivative factors from the massless propagator $\left(k_{1} \cdot k_{2}\right)^{-1}$ is the same as in the open string scattering. So the number of $k$ 's in the numerator is always equal or greater than the number of $k$ 's in the denominator. For terms involving the small $\lambda$ regulator, the analysis of inverse derivative factors is more complicated and will not be attempted in this paper.

The previous paragraph clarifies a statement in [3] concerning graviton scattering in type II theories. In that case, integration over fermionic and bosonic zero modes in fourgraviton scattering at genus $g$ led to a prefactor in the low-energy amplitude of the form 


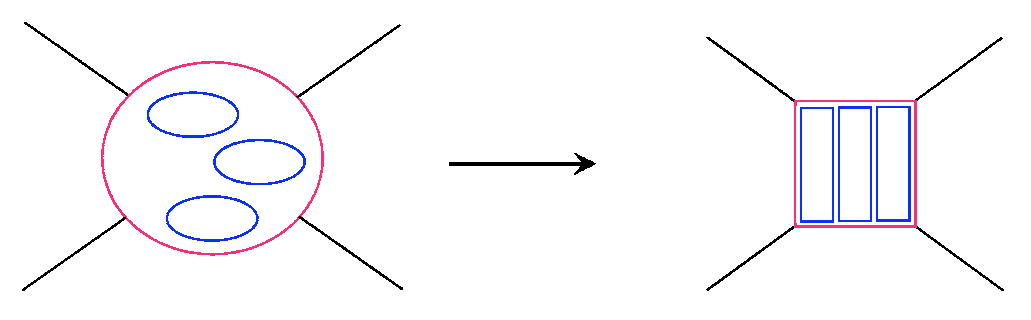

Figure 1. Three-loop contribution to the single-trace term in four-gluon scattering and its connection to the $\operatorname{tr} F^{4}$ term in Yang-Mills theory in the low energy limit.

$s^{g} R^{4} I(s, t, u)$ for $g \leq 6$, multiplying a complicated dynamical function of the external momenta. The conclusion that these terms are $F$-terms relied on the absence of inverse powers of $s$ in $I(s, t, u)$ arising from potential closed-string poles in the dynamical factor multiplying the zero-mode prefactor. The arguments of the previous paragraph verify that no such inverse powers are present and these terms are indeed $F$-terms, at least up to $L=5$ where the complicated small $\lambda$ regulator is unnecessary. This lends support to the arguments in [4] that ultraviolet divergences are absent in $\mathcal{N}=8$ supergravity up to at least eight loops.

\section{Contributions of handles}

Open superstring theory is well-known to generate a closed-string, or gravitational, sector in string perturbation theory, starting at one loop $(L=1)$. In order to decouple gravity in the low energy limit one needs to take the large- $N$ limit, where each additional handle is suppressed by a power of $1 / N^{2}$. Nevertheless, it is interesting to consider how the inclusion of handles in the world-sheet computation at finite $N$ (and the resulting coupling to closed string modes) affects the $t_{8} \operatorname{tr}\left(F^{4}\right)$ and $t_{8}\left(\operatorname{tr} F^{2}\right)^{2}$ terms in the low-energy superstring effective action.

We will now suppose the world-sheet has $H$ handles and $B$ boundaries. The open string amplitude with $M$ vertex operators on a world-sheet of given topology carries a factor $g_{s}^{-\chi}$, where $\chi=2-2 H-B$ is the Euler number (where we again set the number of crosscaps $C=0$ as appropriate for oriented strings). We are particularly interested in the case $M=4$. Note that each handle is associated with a power $g_{s}^{2}$, which is equivalent to the insertion of two boundaries, but whereas each free boundary generates a factor $N$, a handle does not depend on $N$. Each handle is associated with three complex moduli and bring three $b$-ghosts and their complex conjugates, so a world-sheet with $H$ handles and $B$ boundaries has $3(2 H+B-2) b$-ghost insertions. The four-gluon amplitude on a world-sheet with $L=B+2 H-1$ boundaries and $H$ handles is a simple generalization of (2.2) and (2.3), with each handle counting as two boundaries and an appropriate change in the power of $N$ as described above.

Figure 1 illustrates a contribution to the single-trace amplitude with three loops and no handles, $H=0, B=4$, and an example of a Feynman diagram that arises in the low energy planar Yang-Mills limit. Figure 2 illustrates a contribution of the same order in string coupling that has one handle, $H=1, B=2$. Two distinct boundaries of moduli space contribute in the low energy field theory limit. One of these arises from the short handle limit, 

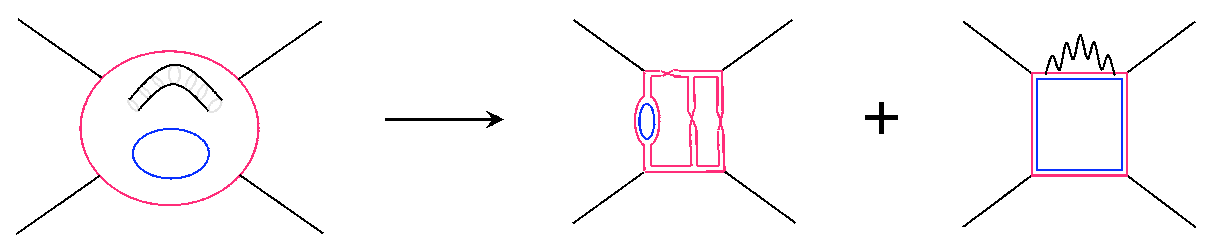

Figure 2. A world-sheet with one handle and two boundaries that contributes to the single-trace term at the same order as the four-boundary amplitude of figure 1. The figure illustrates examples of field theory diagrams that arise from the short-handle and long-handle boundaries of moduli space in the low energy limit.

which contributes a sum of three-loop non-planar Yang-Mills diagrams, which give contributions analogous to those of figure 1, but with two fewer powers of $N$. The other arises from the limit in which the handle is long and picks out the ground state graviton exchange, resulting in a sum of Yang-Mills loop diagrams with a graviton propagator attached.

The first example where a handle contributes is the addition of a handle $(H=1)$ to the tree-level disk world-sheet - i.e., to the world-sheet with $B=1$. This is associated with a factor of $g_{s} s t_{8} \operatorname{tr} F^{4}$ in the low energy limit, whereas the two-loop $(B=3, H=0)$ $t_{8} \operatorname{tr} F^{4}$ term has a factor of $g_{s} N^{2} s t_{8} \operatorname{tr} F^{4}$. So to order $g_{s}$, the analytic contribution to the low-energy expansion of the four-point open string amplitude is given by

$$
\mathcal{A}_{L=2}^{\text {ana. }} \sim g_{s} \quad\left(\left(c_{3,0} N^{2}+c_{1,1}\right) s t_{8} \operatorname{tr}\left(F^{4}\right)+d_{3,0} N s t_{8}\left(\operatorname{tr} F^{2}\right)^{2}\right),
$$

where $c_{B, H}$ and $d_{B, H}$ are coefficients which in principle could be computed. There is no canonical way of separating the open-string and closed contributions to the $c_{1,1}$ coefficient which is subleading in $1 / N^{2}$. We will return to this point in the next section when we will discuss the connection with the pure field theoretical super-Yang-Mills results of [23, 24, 29].

With $L=3$ the $B=4, H=0$ term is accompanied by a contribution with $B=2$, $H=1$, which is a $1 / N^{2}$ correction. In this case the leading contribution to the analytic part of the low-energy expansion of the three-loop four-point open string amplitude is given by

$$
\mathcal{A}_{3}^{\text {ana. }} \sim g_{s}^{2}\left(\left(c_{4,0} N^{3}+c_{2,1} N\right) s t_{8} \operatorname{tr}\left(F^{4}\right)+\left(d_{4,0} N^{2}+d_{2,1}\right) s^{2} t_{8}\left(\operatorname{tr} F^{2}\right)^{2}\right),
$$

where the double-trace term is proportional to $s^{2}$ because of the absence of inverse derivative factors as described earlier in section 3.2.

With $L=4$ the $B=5, H=0$ term is accompanied by the contributions of a worldsheet with $B=3, H=1$ and $B=1, L=2$ for both the single trace and the double trace terms. In this case the leading contribution to the analytic part of the low-energy expansion of the three-loop four-point open string amplitude is given by

$$
\mathcal{A}_{4}^{\text {ana. }} \sim g_{s}^{3}\left(\left(c_{5,0} N^{4}+c_{3,1} N^{2}+c_{1,2}\right) s t_{8} \operatorname{tr}\left(F^{4}\right)+\left(d_{5,0} N^{3}+d_{3,1} N\right) s^{2} t_{8}\left(\operatorname{tr} F^{2}\right)^{2}\right) .
$$

\section{Connections with maximal SYM theory in various dimensions}

We will now discuss the connections between the low energy limit of open-string results with multi-loop amplitudes in maximally supersymmetric Yang-Mills field theory in $D$ 
dimensions. We are considering the open string theory on $N$ coincident $D p$-branes where $p=D-1$. If the corresponding low energy $\mathrm{U}(N)$ Yang-Mills theory coupling constant (which has non-zero dimension when $D>4$ ) is fixed so that

$$
g_{\mathrm{YM}}^{2}=g_{s} l_{s}^{D-4}=\mathrm{constant}
$$

as $l_{s} \rightarrow 0$, the gravitational coupling vanishes,

$$
\kappa^{2}=g_{s}^{2} l_{s}^{8}=g_{\mathrm{YM}}^{4} l_{s}^{16-2 D} \rightarrow 0,
$$

provided $D<8$ (or $p<7$ ), which is the condition that the gravitational back reaction of the $D p$-brane can be ignored.

As mentioned earlier, although we are decoupling the closed-string sector, world-sheet handles are nevertheless expected to make a contribution to the theory in the low energy limit. In section 4 we discussed the effect of handles on the low-energy expansion of the open string amplitudes and we saw that there is no way of separating 'open string' contributions from 'closed string' contributions to the sub-leading $1 / N^{2}$ corrections. One way to see this is to consider, for instance, the following contribution to the two-loop effective action from the four-point amplitude

$$
S_{L=2}=\int d^{D} x \sqrt{-g} g_{s} l_{s}^{10-D}\left(c_{3,0} N^{2}+c_{1,1}\right) \partial^{2} t_{8} \operatorname{tr}\left(F^{4}\right) .
$$

Using the relations (5.1) and (5.2) one can write the $1 / N^{2}$ correction to the effective action either as a super-Yang-Mills contribution $c_{1,1} g_{\mathrm{YM}}^{2} l_{s}^{2(7-D)} \partial^{2} t_{8} \operatorname{tr}\left(F^{4}\right)$ or as a mixed Yang-Mills and gravity contribution $c_{1,1} \kappa l_{s}^{6-D} \partial^{2} t_{8} \operatorname{tr}\left(F^{4}\right)$. Because there is no way of (and no meaning in) separating the gravitational contribution from the super-Yang-Mills in string theory, we will only focus on the large $N$ contribution by restricting our attention to the terms of order $N^{L}$ and $N^{L-1}$, which get no contribution from world-sheet handles.

\subsection{Onset of ultraviolet divergences in various dimensions}

In the limit $l_{s} \rightarrow 0$ the string theory results have clear implications for the structure of the ultraviolet divergences of the four-gluon SYM field theory calculations. In particular, we see why the single trace term has worse ultraviolet divergences than the double-trace term. A clear way of characterising this is by determining the "critical dimension", $D_{c}$, which is the minimal dimension in which a $L$-loop term diverges in the ultraviolet - i.e., the dimension in which the ultraviolet divergence is logarithmic.

To begin, we note that the superficial degree of divergence of a $L$-loop Feynman diagram contribution to the four-gluon amplitude in $D$ dimensions is $\Lambda^{(D-4) L}$, where $\Lambda$ is a momentum cut-off. Since there is also a prefactor of $t_{8} F^{4}$ at all orders, the divergence is reduced to $\Lambda^{(D-4) L-4}$. However, in the case of the single trace term we found that there is a factor of $g_{s}^{L-1} \partial^{2} t_{8} \operatorname{tr} F^{4}$ for $1<L \leq 5$, so that the degree of divergence is $\Lambda^{(D-4) L-6}$. We therefore reproduce the result that the single-trace term is ultraviolet finite in dimensions satisfying $D<D_{c}=4+6 / L$, at least up to $L=5$ and quite probably for all $L$. In these dimensions the amplitude has a negative mass dimension indicating the presence of infrared 


\begin{tabular}{|cccccc|}
\hline & $\mathrm{L}=1$ & $\mathrm{~L}=2$ & $\mathrm{~L}=3$ & $\mathrm{~L}=4$ & $\mathrm{~L}=5$ \\
\hline$\partial^{2 \gamma_{L}} t_{8} \operatorname{tr}\left(F^{4}\right)$ & $D_{c}=8$ & $D_{c}=7$ & $D_{c}=6$ & $D_{c}=11 / 2$ & $D_{c}=26 / 5$ \\
& $\gamma_{1}=0$ & $\gamma_{2}=1$ & $\gamma_{3}=1$ & $\gamma_{4}=1$ & $\gamma_{5}=1$ \\
\hline$\partial^{2 \beta_{L}} t_{8}\left(\operatorname{tr} F^{2}\right)^{2}$ & $D_{c}=8$ & $D_{c}=7$ & $D_{c}=20 / 3$ & $D_{c}=6$ & $D_{c}=28 / 5$ \\
& $\beta_{1}=0$ & $\beta_{2}=1$ & $\beta_{3}=2$ & $\beta_{4}=2$ & $\beta_{5}=2$ \\
\hline
\end{tabular}

Table 1. Critical dimensions for the single trace and double trace contributions at different loop orders.

divergences given by inverse powers of the external momenta. In the case of the $L$-loop contribution to the double-trace term the prefactor has the form $g_{s}^{L-1} \partial^{2\lceil L / 2\rceil} t_{8}\left(\operatorname{tr} F^{2}\right)^{2}$ so that the degree of divergence is, for $1<L \leq 4, \Lambda^{(D-4) L-2\lceil L / 2\rceil-4}$. For this range of $L$ the amplitude is ultraviolet finite in dimensions satisfying $D<D_{c}=4+(4+2\lceil L / 2\rceil) / L$. Although we have no firm statements at higher loops, we expect that since $\partial^{4} t_{8}\left(\operatorname{tr} F^{2}\right)^{2}$ is a $D$-term it will receive corrections from all $L \geq 5$. It would then follows that for $L \geq 3$ there are no ultraviolet divergences in $D<D_{c}=4+8 / L$. In these dimensions the double trace contribution to the amplitude has a negative mass dimension, again indicating the presence of infrared divergences represented by inverse powers of the external momenta. The results are summarised in the table 1 below and match the field theory results of $[23,24,29]$ for the evaluation of the four gluon amplitude up to $L=4$.

Although there is no canonical way of separating the gravitational contributions from the pure Yang-Mills corrections in string theory, the $1 / N^{2}$ corrections described in section 4 qualitatively reproduce the result quoted in [24] with the exception of the absence of a contribution independent of $N$ to the $\partial^{2} t_{8} \operatorname{tr} F^{4}$ counterterm at four loops in $D=11 / 2$.

\section{Summary and comments on higher-point amplitudes}

In this paper, we have analyzed open superstring four-point amplitudes using the pure spinor formalism and determined non-renormalization properties of certain terms in the low-energy effective action. Terms in the effective action proportional to $t_{8} \operatorname{tr}\left(F^{4}\right)$ and $t_{8}\left(\operatorname{tr} F^{2}\right)^{2}$ were shown not to receive corrections above one-loop, as expected from their connection to the anomaly-cancelling term $B \wedge F^{4}$. Furthermore, the $\partial^{2} t_{8}\left(\operatorname{tr} F^{2}\right)^{2}$ term was shown to not receive corrections above two loops. On the other hand, the $\partial^{4} t_{8}\left(\operatorname{tr} F^{2}\right)^{2}$ and $\partial^{2} t_{8} \operatorname{tr}\left(F^{4}\right)$ terms are expected to receive corrections to all loops. These statements were verified up to five loops using the pure spinor prescription for the four-point amplitudes.

This behaviour can be heuristically explained using supersymmetry arguments based on $F$-terms and $D$-terms. The terms $t_{8} \operatorname{tr}\left(F^{4}\right), t_{8}\left(\operatorname{tr} F^{2}\right)^{2}$ and $\partial^{2} t_{8}\left(\operatorname{tr} F^{2}\right)^{2}$ are $F$-terms which are expected to satisfy non-renormalization conditions. For $\partial^{2} t_{8} \operatorname{tr}\left(F^{4}\right)$ and $\partial^{4} t_{8}\left(\operatorname{tr} F^{2}\right)^{2}$ the behavior is different since when $D<10, \partial^{2} t_{8} \operatorname{tr}\left(F^{4}\right)$ can be written as the $D$-term, $\int d^{16} \theta \operatorname{tr}(\varphi \varphi)$, where $\varphi$ is a non-linear superfield whose lowest component is a scalar, and $\partial^{4} t_{8}\left(\operatorname{tr} F^{2}\right)^{2}$ can be expressed in terms of $\int d^{16} \theta \operatorname{tr}(\varphi \varphi)^{2}$ and $\int d^{4} x \int d^{16} \theta T^{2}$ where $T_{A B}$ is the symmetric traceless supercurrent [26]. So $\partial^{2} t_{8} \operatorname{tr}\left(F^{4}\right)$ and $\partial^{4} t_{8}\left(\operatorname{tr} F^{2}\right)^{2}$ are not expected to satisfy any non-renormalization conditions. In the analysis of open superstring ampli- 
tudes, the $t_{8}\left(\operatorname{tr} F^{2}\right)^{2}$ and $t_{8} \operatorname{tr}\left(F^{4}\right)$ terms behave differently since inverse derivative factors from colliding vertex operators are present in the $t_{8} \operatorname{tr}\left(F^{4}\right)$ computation but are absent in the $t_{8}\left(\operatorname{tr} F^{2}\right)^{2}$ computation. It would be useful to better understand the relation between these inverse derivative factors and the nonlinear construction of the $D$-term $\int d^{16} \theta \operatorname{tr}(\varphi \varphi)$.

Our analysis of $t_{8} \operatorname{tr}\left(F^{4}\right)$ and $t_{8}\left(\operatorname{tr} F^{2}\right)^{2}$ terms is consistent with the field theory computations of $[23,24,29]$ and explains the apparent puzzle that $t_{8}\left(\operatorname{tr} F^{2}\right)^{2}$ terms are less divergent in the ultraviolet than $t_{8} \operatorname{tr}\left(F^{4}\right)$ terms. In addition, our analysis showed that there are no inverse derivative factors in the analogous Type II computation, at least for terms which do not require the small $\lambda$ regulator. This lends support to the previous claim of [3] that for $g<6, \partial^{2 g} R^{4}$ terms do not receive contributions above genus $g$ and to the arguments in [4] that ultraviolet divergences are absent in four-dimensional $\mathcal{N}=8$ supergravity up to at least eight loops. Therefore, the first "surprise" would arise if the four-graviton amplitude was not ultraviolet divergent at nine loops in four dimensions.

It would be very interesting to generalize the methods of this paper to higher-point amplitudes beyond four points. Since higher-point amplitudes have massless poles, one needs to first subtract out the massless poles before using the amplitudes to determine non-renormalization properties of terms in the low-energy effective action. At the moment, it is unclear how to verify that subtracting out the massless poles does not affect the nonrenormalization properties implied by the zero-mode counting. Nevertheless, one expects that certain higher-point terms in the effective action will satisfy non-renormalization conditions and one can make some preliminary speculations on how the behavior of $F^{4}$ terms extend to $F^{n}$ terms. In particular, the extension of our analysis of the zero mode saturation to five-point amplitudes indicates that the $\operatorname{tr} F^{5}, \operatorname{tr} F^{3} \times \operatorname{tr} F^{2}$ should be one-loop exact, that the $\partial^{2} \operatorname{tr} F^{5}$ and $\partial^{2}\left(\operatorname{tr} F^{3}\right)\left(\operatorname{tr} F^{2}\right)$ should be two-loop exact, while $\partial^{4} F^{5}$ are $D$-terms and should get contributions to all loops for all group theory structures. For the six-point amplitude, the zero mode saturation indicates that the $\operatorname{tr} F^{6},\left(\operatorname{tr} F^{3}\right)^{2}$ and $\left(\operatorname{tr} F^{2}\right)^{3}$ should be one-loop exact, while $\partial^{2} F^{6}$ should receive perturbative contributions to all orders for all group theory structures.

\section{Acknowledgments}

We would like to thank Zvi Bern, Kelly Stelle, Radu Roiban and Edward Witten for discussions. The research of NB was partially supported by CNPq grant 300256/94-9 and FAPESP grant 04/11426-0. The research of PV was supported in part by the ANR grant BLAN06-3-137168. JGR acknowledges support by research grants MCYT FPA 2007-66665, 2009SGR502.

\section{References}

[1] N. Berkovits, Pure spinor formalism as an $N=2$ topological string, JHEP 10 (2005) 089 [hep-th/0509120] [SPIRES].

[2] N. Berkovits and N. Nekrasov, Multiloop superstring amplitudes from non-minimal pure spinor formalism, JHEP 12 (2006) 029 [hep-th/0609012] [SPIRES]. 
[3] N. Berkovits, New higher-derivative $R^{4}$ theorems, Phys. Rev. Lett. 98 (2007) 211601 [hep-th/0609006] [SPIRES].

[4] M.B. Green, J.G. Russo and P. Vanhove, Ultraviolet properties of maximal supergravity, Phys. Rev. Lett. 98 (2007) 131602 [hep-th/0611273] [SPIRES].

[5] P.S. Howe and K.S. Stelle, Supersymmetry counterterms revisited, Phys. Lett. B 554 (2003) 190 [hep-th/0211279] [SPIRES].

[6] G. Bossard, P.S. Howe and K.S. Stelle, The ultra-violet question in maximally supersymmetric field theories, Gen. Rel. Grav. 41 (2009) 919 [arXiv:0901.4661] [SPIRES].

[7] S. Mandelstam, Light cone superspace and the ultraviolet finiteness of the $N=4$ model, Nucl. Phys. B 213 (1983) 149 [SPIRES].

[8] L. Brink, O. Lindgren and B.E.W. Nilsson, The ultraviolet finiteness of the $N=4$ Yang-Mills theory, Phys. Lett. B 123 (1983) 323 [SPIRES].

[9] P.S. Howe, K.S. Stelle and P.C. West, A class of finite four-dimensional supersymmetric field theories, Phys. Lett. B 124 (1983) 55 [SPIRES].

[10] P.S. Howe, K.S. Stelle and P.K. Townsend, The relaxed hypermultiplet: an unconstrained $N=2$ superfield theory, Nucl. Phys. B 214 (1983) 519 [SPIRES].

[11] M.B. Green, J.H. Schwarz and E. Witten, Superstring theory. Vol. 2: loop amplitudes, anomalies and phenomenology, Cambridge Monographs On Mathematical Physics, Cambridge University Press Cambridge U.K. (1987).

[12] E.A. Bergshoeff and M. de Roo, The quartic effective action of the heterotic string and supersymmetry, Nucl. Phys. B 328 (1989) 439 [SPIRES].

[13] M. de Roo, H. Suelmann and A. Wiedemann, The supersymmetric effective action of the heterotic string in ten-dimensions, Nucl. Phys. B 405 (1993) 326 [hep-th/9210099] [SPIRES].

[14] A.A. Tseytlin, On $\mathrm{SO}(32)$ heterotic-type-I superstring duality in ten dimensions, Phys. Lett. B 367 (1996) 84 [hep-th/9510173] [SPIRES].

[15] A.A. Tseytlin, Heterotic-type-I superstring duality and low-energy effective actions, Nucl. Phys. B 467 (1996) 383 [hep-th/9512081] [SPIRES].

[16] C. Bachas, C. Fabre, E. Kiritsis, N.A. Obers and P. Vanhove, Heterotic-type-I duality and D-brane instantons, Nucl. Phys. B 509 (1998) 33 [hep-th/9707126] [SPIRES].

[17] S. Stieberger and T.R. Taylor, Non-Abelian Born-Infeld action and type-I-heterotic duality. II: nonrenormalization theorems, Nucl. Phys. B 648 (2003) 3 [hep-th/0209064] [SPIRES].

[18] Z.-J. Zheng, J.-B. Wu and C.-J. Zhu, Two-loop superstrings in hyperelliptic language. I: the main results, Phys. Lett. B 559 (2003) 89 [hep-th/0212191] [SPIRES]; Two-loop superstrings in hyperelliptic language. II: the vanishing of the cosmological constant and the non- renormalization theorem, Nucl. Phys. B 663 (2003) 79 [hep-th/0212198] [SPIRES]; Two-loop superstrings in hyperelliptic language. III: the four-particle amplitude, Nucl. Phys. B 663 (2003) 95 [hep-th/0212219] [SPIRES].

[19] E. D'Hoker and D.H. Phong, Two-loop superstrings VI: non-renormalization theorems and the 4-point function, Nucl. Phys. B 715 (2005) 3 [hep-th/0501197] [SPIRES].

[20] N. Berkovits and C.R. Mafra, Equivalence of two-loop superstring amplitudes in the pure spinor and RNS formalisms, Phys. Rev. Lett. 96 (2006) 011602 [hep-th/0509234] [SPIRES]. 
[21] N. Berkovits, Super-Poincaré covariant two-loop superstring amplitudes, JHEP 01 (2006) 005 [hep-th/0503197] [SPIRES].

[22] Z. Bern, L.J. Dixon, D.C. Dunbar, M. Perelstein and J.S. Rozowsky, On the relationship between Yang-Mills theory and gravity and its implication for ultraviolet divergences, Nucl. Phys. B 530 (1998) 401 [hep-th/9802162] [SPIRES].

[23] Z. Bern, J.J.M. Carrasco, H. Johansson and D.A. Kosower, Maximally supersymmetric planar Yang-Mills amplitudes at five loops, Phys. Rev. D 76 (2007) 125020 [arXiv: 0705.1864] [SPIRES].

[24] L. Dixon, Multi-loop amplitudes with maximal supersymmetry, talk given at the meeting International workshop on gauge and string amplitudes, IPPP, Durham U.K. March 30April 3 2009;

Z. Bern, J.J. M. Carrasco, L. Dixon, R. Roiban and H. Johansson, to appear.

[25] K. Peeters, P. Vanhove and A. Westerberg, Supersymmetric higher-derivative actions in ten and eleven dimensions, the associated superalgebras and their formulation in superspace, Class. Quant. Grav. 18 (2001) 843 [hep-th/0010167] [SPIRES].

[26] J.M. Drummond, P.J. Heslop, P.S. Howe and S.F. Kerstan, Integral invariants in $N=4$ SYM and the effective action for coincident D-branes, JHEP 08 (2003) 016 [hep-th/0305202] [SPIRES].

[27] Y. Aisaka and N. Berkovits, Pure spinor vertex operators in Siegel gauge and loop amplitude regularization, JHEP 07 (2009) 062 [arXiv: 0903.3443] [SPIRES].

[28] P.A. Grassi and P. Vanhove, Higher-loop amplitudes in the non-minimal pure spinor formalism, JHEP 05 (2009) 089 [arXiv:0903.3903] [SPIRES].

[29] Z. Bern, J.S. Rozowsky and B. Yan, Two-loop four-gluon amplitudes in $N=4$ super-Yang-Mills, Phys. Lett. B 401 (1997) 273 [hep-ph/9702424] [SPIRES]. 